\title{
Absence of Pair Binding in the $U=\infty$ Hubbard Model
}

\author{
S. CHAKRAVARTY', L. CHAYES ${ }^{2}$, and S. A. KIVELSON ${ }^{\prime}$ \\ ${ }^{1}$ Department of Physics, University of California, Los Angeles, CA 90024, U.S.A. \\ 'Departmem of Mathematics, University of California, Los Angeles, CA 90024, U.S.A.
}

(Reccived: 17 September 1991; revised version 15 October 1991)

\begin{abstract}
We consider the standard Hubbard model in the $U=\infty$ limit. We show that, for any finite lattice with all positive hopping matrix elements, $t_{t, 5}>0$, the ground state energy of the system containing two particles in excess of half filling plus the energy of the system at half filling is never lower than twice the energy of the system with a single extra particle. Similar results are obtained for 'holes' when the lattice is bipartite. As a byproduct, we abtain a simple alternative proof of Tasaki's generalization of the Nagaoka theorem for non-bipartite lattices (but without the uniqueness clause).
\end{abstract}

AMS sobject classification (1991). 82B20.

It is of considerable interest to investigate the question of how, in the presence of purely repulsive electron-electron interactions, many body effects can produce an effective attraction between electrons or holes. For instance, a recent theory of superconductivity in doped $C_{60}$ proposes that an effective force between conduction band electrons arises from an overscreening of the purely repulsive microscopic interaction between electrons on the $C_{60}$ molecule [1].

Here, we consider the problem of adding or subtracting a few electrons to a finite size Hubbard model at half filling. We assume that such models can capture the essential features of 'molecules': Electron-electron interactions are represented in as simple a way as possible via the Hubbard interaction.

We focus principally on the problem of adding electrons rather than the more commonly considered problem of adding holes; it has been determined that $C_{60}$ can be electron doped but, at present, it has not been hole doped. A molecule will be deemed 'neutral' when, for $N$ sites, there are exactly $N$ electrons and it will be called singly charged if there are $N+1$ electrons, etc. We denote the ground-state energy of the $n$-fold charged molecule by $E_{n}$. The pair binding energy for two electrons is therefore defined as

$$
\Lambda_{\varepsilon}=2 E_{1}-E_{2}-E_{0}
$$

Using similar notation for the system which is $n$ electrons below half filling (i.e. has $n$ 'holes'), we define

$$
\Delta_{\mathrm{h}}=2 E_{-1}-E_{-2}-E_{0}
$$


to be the binding energy for two holes. Thus, when $\Delta_{e}$ is positive, it is energetically preferable for a system to place two extra electrons on a single molecule and leave a second molecule neutral than to put a single electron on each of two molecules. In this sense, a positive $\Delta_{\varepsilon}$ represents an effective attraction between the two electrons. Similar considerations may be applied to the hole pairing energy $\Delta_{\mathrm{h}}$.

We define our model on a lattice of $N$ sites each of which contains one or zero electrons of each spin type. The Hubbard Hamiltonian is given by

$$
H=-\sum_{i, j, \sigma} t_{i, j} c_{t, \sigma}^{\dagger} c_{j, \sigma}+U \sum_{i} n_{t, \uparrow} n_{i, \downarrow},
$$

where $n_{i, \sigma}=c_{j, \sigma}^{\dagger} c_{i, \sigma} ; \sigma=\uparrow$ or $\downarrow$ and the $c_{i, \sigma}$ 's obey the usual (fermionic) anti-commutation relations, i.e.

$$
\left\{c_{i, \sigma}, c_{y, \sigma^{\prime}}\right\}=\left\{c_{i, \sigma}^{\dagger}, c_{j, \sigma^{\prime}}^{\dagger}\right\}=0, \quad\left\{c_{i, \sigma}, c_{j, \sigma^{\prime}}^{\dagger}\right\}=\delta_{i, j^{\prime}} \delta_{\sigma, \sigma^{*}}
$$

It is known from exact diagonalization studies of various Hubbard clusters that there are structures where, for certain ranges of the parameter $U$, the $\Delta$ 's in Equation (1) are positive [2]. Now for $U=0$, it is readily established that $\Delta \leqslant 0$. In this Letter, we consider the $U=\infty$ limit and show that under quite general circumstances, both $\Delta_{\mathrm{e}} \leqslant 0$ and $\Delta_{\mathrm{h}} \leqslant 0$. Thus, pair binding is an effect which occurs only at 'intermediate' energies and the pair binding energies, $\Delta_{\mathrm{c}}$ and $\Delta_{\mathrm{h}}$ are likely to be somewhat complicated functions of $U$. (For example, when a structure does enjoy pair binding, the associated $\Delta$ cannot be a monotone function of $U$.)

To exhibit the $U=\infty$ behavior of (2), we subtract from the Hamiltonian $[U] \times[$ a term which counts the minimum possible number of doubly occupied sites]. Specifically, in the $n$-excess particle sector, we subtract off $n U$ for $n \geqslant 0$ and do nothing if $n \leqslant 0$. After this subtraction, the coefficient of $U$ now counts the number of unnecessary doubly occupied sites in the system. Let $\mathscr{P}\left(\equiv \mathscr{P}_{n}\right)$ denote the projection operator which selects only those states that have no unnecessary double occupations. For the large $U$ limit, the sensible term to consider is the Hamiltonian:

$$
\mathscr{H}=\mathscr{P}\left[-\sum_{i, \sigma, \sigma} t_{i, \lambda} c_{t, \sigma}^{\dagger} c_{,, \sigma}\right] \mathscr{P} .
$$

It is not hard to demonstrate (recall that $N<\infty$ ) that the corrections to those eigenfunctions of $\mathscr{H}$ residing in the projected subspace and their associated eigenvalues cannot exceed the order of $1 / U$.

In our discussion of the Hamiltonian (4), it should be mentioned that, because we are considering only time reversal invariant systems, the $t_{h, j}$ are real. Furthermore, Hermiticity, requires that the $t_{t, j}$ are symmetric: $t_{t, y}=t_{j, l}$. We consider the case in which all the diagonal elements, $t_{i, l}$ are equal (and, hence, can be set to zero without loss of generality). We will be primarily interested in the systems which do not have any negative $t_{i,}$. This is a natural assumption which accurately describes the single electron physics when the inter-atomic orbital overlap is positive (as will be the case for $\pi$-electrons in a planar cluster) and pairwise small compared with unity. 
In the case of electrons, we will make no additional assumptions. However, for holes, we will be forced to assume that the lattice is bipartite, which means that the lattice can be divided into two disjoint collections of sites - 'red' and 'black' - and that the $t_{i, J}$ are nonzero only when they connect sites of opposite color.

Our principal results stem from the following:

PROPOSITION 1. Let $|\psi\rangle$ be any state with $n \geqslant 0$ and let $q_{i}=\left[\left\langle\psi\left|n_{i, 1} n_{i, 1}\right| \psi\right\rangle\right]^{1 / 2}$ denote the 'excess charge amplitude' at site $i$. Then

$$
Q_{t, \gamma} \equiv\left|\left\langle\psi\left|\mathscr{P}\left[\sum_{\sigma} c_{t, \sigma}^{+} c_{\gamma, \sigma}\right] \mathscr{P}\right| \psi\right\rangle\right| \leqslant q_{t} q_{j} .
$$

PROPOSITION 2. Let $\mu_{1}$ denote the square-root of the probability that the site $i$ is devoid of electrons. Then, for any state $|\psi\rangle$ below half filling,

$$
M_{i, J} \equiv\left|\left\langle\psi\left|\mathscr{P}\left[\sum_{\sigma} c_{l, \sigma}^{\dagger} c_{y, \sigma}\right] \mathscr{P}\right| \psi\right\rangle\right| \leqslant \mu_{\imath} \mu_{j}
$$

Remark. This is not particularly difficult to show because there are only a few possibilities for wave functions which 'survive' the operator $\mathscr{P}\left[\Sigma_{\sigma} c_{t, \sigma}^{+} c_{j, \sigma}\right] \mathscr{P}$. For example, in the cases $n \geqslant 0$, it is clear that whenever $i$ and $j$ are both singly occupied, the result will be zero since $\Sigma_{\sigma} c_{t, \sigma}^{\dagger} c_{j, \sigma}$ - at best - creates an unnecessary hole.

Proof of Proposition 1. Let us write, in all possible generality, a state with $n \geqslant 0$,

$$
\begin{aligned}
& |\psi\rangle=\left[\mathscr{A}_{1}\left(c_{i, \uparrow}^{\dagger} c_{j, \uparrow}^{\dagger}\right)+\mathscr{A}_{2}\left(c_{i, \downarrow}^{+} c_{j, \downarrow}^{\dagger}\right)+\mathscr{A}_{3}\left(c_{i, \uparrow}^{\dagger} c_{i, 1}^{\dagger}\right)+\mathscr{A}_{4}\left(c_{i, \downarrow}^{\dagger} c_{j, \uparrow}^{\dagger}\right)+\right. \\
& +\mathscr{B}_{1}\left(c_{i, \uparrow}^{+} c_{i, \downarrow}^{+} c_{j, 1}^{\dagger}\right)+\mathscr{B}_{2}\left(c_{1, \uparrow}^{\dagger} c_{i, \downarrow}^{\dagger} c_{j, \downarrow}^{\dagger}\right)+ \\
& +\mathscr{C}_{1}\left(c_{j . \uparrow}^{+} c_{j, 1}^{\dagger} c_{t, 1}^{\dagger}\right)+\mathscr{G}_{2}\left(c_{3.1}^{\dagger} c_{j, \downarrow}^{\dagger} c_{t, 1}^{\dagger}\right)+ \\
& \left.+\mathscr{D}\left(c_{i, \uparrow}^{\dagger} c_{r, \downarrow}^{\dagger} c_{r, \uparrow}^{\dagger} c_{i, \downarrow}^{\dagger}\right)\right]|0\rangle,
\end{aligned}
$$

where in the above expression, the operators $\mathscr{A}_{1} \ldots$ operate on coordinates other than $i$ and $j$ and it is assumed that none of these operators are affected by $\mathscr{P}$. (In particular, we have omitted basis states with no electrons on site $i$ or $j$ since these will be annihilated by $\mathscr{P}$.) Note that, by definition,

$$
q_{i}^{2}=\left\langle 0\left|\mathscr{B}_{1}^{\dagger} \mathscr{B} 1+\mathscr{B}_{2}^{\dagger} \mathscr{B}_{2}+\mathscr{D}^{\dagger} \mathscr{Z}\right| 0\right\rangle \equiv\left|\mathbf{B}_{1}\right|^{2}+\left|\mathbf{B}_{2}\right|^{2}+|\mathbf{D}|^{2},
$$

etc. Now. as mentioned in the remark, none of the 'A' terms contribute to $Q_{i,}$, and, for a similar reason, there is no contribution from the $\mathrm{D}$ term (which is anyway absent altogether when $n<2$ ). We are left with

$$
\left|\left\langle\psi\left|\mathscr{P}\left[\sum_{\sigma} c_{r, \sigma}^{\dagger} c_{J, \sigma}\right] \mathscr{P}\right| \psi\right\rangle\right|=\left|\left\langle 0\left|\mathscr{B}_{1}^{+} \mathscr{C}_{1}\right| 0\right\rangle+\left\langle 0\left|\mathscr{B}_{2}^{+} \mathscr{C}_{2}\right| 0\right\rangle\right|
$$

so that

$$
\left|\left\langle\psi\left|\mathscr{P}\left[\sum_{\sigma} c_{r, \sigma}^{\dagger} c_{1, \sigma}\right] \mathscr{P}\right| \psi\right\rangle\right| \leqslant\left|\mathbf{B}_{1}\right|\left|\mathbf{C}_{1}\right|+\left|\mathbf{B}_{2}\right|\left|\mathbf{C}_{2}\right| .
$$


Now

$$
\left.\left|\mathbf{B}_{1}\right|\left|\mathbf{C}_{1}\right|+\left|\mathbf{B}_{2}\right|\left|\mathbf{C}_{2}\right| \leqslant\left(\left|\mathbf{B}_{1}\right|^{2}+\left|\mathbf{B}_{2}\right|^{2}\right)^{1 / 2}\left(\left|\mathbf{C}_{1}\right|^{2}\right)+\left|\mathbf{C}_{2}\right|^{2}\right)^{1 / 2}
$$

which implies the desired inequality, Proposition 1.

The proof of Proposition 2 follows exactly the steps of Proposition 1 so we will omit the details.

In order to properly state our first theorem, we need to introduce a bit of notation. For the collection of the $\left(t_{i, j}\right) \equiv \mathrm{t}$ which go into the make-up of the Hamiltonian, consider the associated lattice 'Laplacian' $\Delta_{t}$ (which here is entirely off-diagonal) that acts on scalar functions $\phi_{i}$ via

$$
\left(\Delta_{t} \phi\right)_{i}=\sum_{j} t_{i, j} \phi_{j}
$$

Let $-\varepsilon_{0}$ denote the lowest eigenvalue of the operator $-\Delta_{r}$. Then we have

THEOREM 1. For $t_{i, j} \geqslant 0$ and $n \geqslant 0, E_{n} \geqslant-n c_{0}$.

Proof. As is well known, $-\varepsilon_{0}$ is obtained by finding the $\phi$ which minimizes

$$
-\left(\phi \Delta_{i} \phi\right)=-\sum_{i, l} \bar{\phi}_{i} t_{i, j} \phi_{J}
$$

subject to the constraint that the $\left|\phi_{i}\right|^{2}$ add up to one. When none of the $t_{i, 3}$ are negative, we get that

$$
\langle\psi|\mathscr{H}| \psi\rangle \geqslant-\sum_{s, j} q_{i} t_{i, j} q_{j}
$$

so that $q_{i}$ may be regarded as a trial function - whose square sums to $n$ - for the operator $-\Delta_{t}$. Theorem 1 follows immediately.

COROLLARY. In the $U=\infty$ limit, $\Delta_{\mathrm{e}} \leqslant 0$.

Proof. It is immediately obvious that $E_{0}=0$. Starting from the bound of Theorem 1 , we observe that, for $n=1$, the lower bound can be realized in the ferromagnetic (Nagaoka) states [3,4], that is, the states with the maximum possible spin consistent with the Pauli principle. This gives us the desired inequality.

Remark. In the original references $[3,4]$, it was established that in the one-hole sector, the ferromagnetic states are the unique ground-states of a large class of bipartite lattices. This is referred to as Nagaoka's Theorem. Recently, Tasaki [5] has generalized the theorem to include 'connected' but nonbipartite lattices with all negative hopping matrix elements, $t_{i, j} \leqslant 0$. (As shown below, this is equivalent to the case of one added electron with the $t_{i, j} \geqslant 0$.) The following is a simpler demonstration of the existence of these ground-states. Let $\left|F_{\uparrow}\right\rangle$ denote the ferromagnetic (up) vacuum:

$$
\left|F_{\uparrow}\right\rangle=\mathscr{F}_{i}|0\rangle \equiv \prod_{i} c_{i, \uparrow}^{+}|0\rangle,
$$


where the operators on the right-hand side of (12) are written in some definite order. We define

$$
|i\rangle=c_{i, \downarrow}^{\dagger}\left|F_{\dagger}\right\rangle \text {. }
$$

and observe that, for $j \neq k$,

$$
\left[c_{i, \uparrow}^{\dagger} c_{k, \uparrow}+c_{t, \downarrow}^{\dagger} c_{k, \downarrow}\right]|j\rangle=0 \text {. }
$$

Indeed $c_{i, \uparrow}^{\dagger}$ after anticommuting with $c_{k, \uparrow} c_{j, 1}^{\dagger}$, always destroys the ferromagnetic vacuum, while (since $j \neq k$ ), $c_{k, \downarrow}$ anticommutes through all preceding operators and then destroys the (true) vacuum. On the other hand, if $j=k, c_{j, \downarrow} c_{j, \downarrow}^{\dagger}$ acts like one on the vacuum and we are left with $c_{t, \downarrow}^{\dagger}\left|F_{\dagger}\right\rangle \equiv|i\rangle$. Thus, we have a family of wave functions $(|i\rangle)$ for which

$$
\mathscr{H}|i\rangle=\sum_{j}-t_{i, J}|j\rangle
$$

and from which the existence of the Nagaoka states follows immediately. In particular, if $\phi(i)$ is any eigenfunction of $-\Delta_{t}$, so that $\Sigma_{l} \phi(i) c_{i, f}^{\dagger}|0\rangle$ is a single particle wave function, then

$$
\left|\phi ; F_{\uparrow}\right\rangle=\sum_{1} \phi(i) c_{t, \downarrow}^{\dagger}\left|F_{\uparrow}\right\rangle
$$

will be an eigenfunction with the same energy when the system has a single electron in excess of half filling. Since the lowest energy eigenfunction of this type saturates the bound in Theorem 1, it is necessarily a ground-state.

As a consequence of Proposition 2, we have the analog of Theorem 1, namely that the energy of $n$ holes is no smaller than $-n \varepsilon_{0}$. The difference between particles and holes shows up when we try to construct the Nagaoka states. Indeed, the natural proposal for a state with a hole at $i$ would be

$$
\left|i^{0}\right\rangle \equiv c_{t \uparrow \uparrow}\left|F_{\uparrow}\right\rangle
$$

However, it is straightforward to see that

$$
c_{r, \uparrow}^{\dagger} c_{\lambda, t}\left|i^{0}\right\rangle=-\left|j^{0}\right\rangle
$$

and there is very little that can be done about the minus sign in the general case.

There are, however, two circumstances where the above situation can be turned around: The first is the case in which all the $t_{t, j}$ are nonpositive. In that case, Equation (18) is exactly what is desired - however, the trouble will reemerge when the system is above half filling. This is the problem that was considered by Tasaki. Although such circumstances could arise through a complicated many-body effect in the atomic wavefunctions, it seems a bit unlikely. The second case, which is of somewhat more interest, is when the lattice is bipartite. Here, one can redefine the states via

$$
\left|i^{\not}\right\rangle=\omega(i)\left|i^{0}\right\rangle
$$


where $\omega(i)$ is, for example, +1 if $i$ is on the black sublattice and -1 if $i$ is on the red. Since, by definition, the $t_{t, j}$ are only nonzero if $i$ and $j$ are of different color, one has the desired relationship

$$
t_{i, j} c_{t, 1}^{\dagger} c_{j, 1}\left|i^{\mathrm{g}}\right\rangle=t_{i, j}\left|j^{\not x}\right\rangle
$$

from which the analog of the corollary to Theorem A holds. All in all, this leaves us with

COROLLARY. On a bipartite lattice with all $t_{k, j}$ of the same sign, and $U=\infty$,

$$
\Delta_{\mathrm{e}}, \Delta_{\mathrm{h}} \leqslant 0 \text {. }
$$

\section{Acknowledgements}

We would like to thank David Thouless and Elliott Lieb for various useful conversations. We gratefully acknowledge the Aspen Center for Physics for their hospitality. This work was supported, in part, by The National Science Foundation Grants: DMR-89-07664 (S.C.), DMS-90-09049 (L.C.), and DMR-90-11803 (S.A.K.), all at UCLA. One of us (L.C.) was also partially supported by a grant from the Sloan Foundation.

\section{References}

1. Chrakravarty, S. and Kivelson, S. A., Superconductivity of doped fullerenes, Europhys Lett. 16, $751-754$ (1991).

2. Fano, G., Ortolani, F., and Parola, A., Hole-hole effective interaction in the two-dimensional Hubbard model, Phys. Rev. B42, 6877-6880 (1990);

Parola, A. et al., $d$-Wave, dimer and chiral states in two-dimensional Hubbard models, Phys. Rev. B43, 6190-6193 (1991);

Lin, H. Q., Ground state properties of the two-dimensional Hubbard model, Los Alamos Preprint, 1991;

White, S. R., Chakravarty, S., Gelfand, M. P., and Kivelson, S. A., Pair-binding in small Hubbard molecules, Phys. Rev. B (in press), 1991;

Fye, R. M., Martins, M. J., and Scalettar, R. T., Binding of holes in one-dimensional Hubbard chains, Phys. Rev. B42, 6809-6812 (1990).

3. Nagaoka, Y., Ferromagnetism in a narrow, almost half filled S-band, Phys. Rev. 147, 392-405 (1966).

4. Thouless, D. J., Exchange in solid ${ }^{3} \mathrm{He}$ and the Heisenberg Hamiltonian, Proc. Phys. Soc. 86, 893-904 (1965).

5. Tasaki, H., Extension of Nagaoka's theorem on the large- $U$ Hubbard model, Phys. Rev. B40, 9192-9193 (1989). 